\title{
A case series on successful pregnancy outcomes in patients with Apla syndrome
}

\author{
Priyanka Gaur*, Swati Kochar, Vaibhav Sharma
}

Department of Obstetrics and Gynaecology, Sardar Patel Medical College, Bikaner, Rajasthan, India

Received: 25 June 2021

Revised: 17 July 2021

Accepted: 19 July 2021

\section{*Correspondence:}

Dr. Priyanka Gaur,

E-mail: gaurpriyanka2704@gmail.com

Copyright: ( ) the author(s), publisher and licensee Medip Academy. This is an open-access article distributed under the terms of the Creative Commons Attribution Non-Commercial License, which permits unrestricted non-commercial use, distribution, and reproduction in any medium, provided the original work is properly cited.

\section{ABSTRACT}

The antiphospholipid antibody syndrome (APS) is defined by the persistent presence of antiphospholipid antibodies in patients with recurrent venous or arterial thromboembolism or pregnancy morbidity. Obstetric complications such as recurrent pregnancy loss, oligohydramnios, preterm delivery, fetal growth restriction, preeclampsia, HELLP syndrome and fetal distress are frequently associated with antiphospholipid syndrome. Successful management of pregnancies with APLA syndrome is a huge challenge for treating obstetrician. Obstetric care is based on combined medical and obstetric high risk management and treatment with the association between aspirin and heparin. Here, we present 3 cases of pregnancy with APLA syndrome who were successfully managed and they had uneventful pregnancies with successful outcomes without any complication.

Keywords: APLA syndrome, Aspirin, Heparin

\section{INTRODUCTION}

Antiphospholipid syndrome (APS) is a multisystem disorder diagnosed by the presence of lupus anticoagulant (LAC) and anticardiolipin (aCL) antibodies in association with venous and/or arterial thrombosis or pregnancy complications.

It is an autoimmune thrombophilic condition wherein the antibodies in blood attack the phospholipid binding proteins instead of phospholipid itself. ${ }^{1}$

It can be a discrete entity (primary APS) or it can occur in association with other autoimmune diseases, usually SLE.

Women are more frequently affected (female to male ratio is 5:1) as it is a type of acquired thrombophilia. Antiphospholipid antibodies promote activation of endothelial cells, monocytes and platelets, resulting in excessive production of tissue factor and thromboxane A2. Also, there is an element of complement activation. These factors, along with typical changes in the hemostatic system during normal pregnancy, result in a hypercoagulable state, which in turn result in obstetric complications, including recurrent miscarriage, preterm labor, oligohydramnios, prematurity, FGR, fetal distress, fetal or neonatal thrombosis, pre-eclampsia/eclampsia, HELLP syndrome and placental insufficiency.

A few patients may develop catastrophic antiphospholipid antibody syndrome (CAPS) also called Asherson syndrome characterised by rapid and progressive thromboembolism involving three or more organ systems simultaneously with high maternal mortality. ${ }^{2}$

Treatment with low dose aspirin and low molecular weight heparin can improve the pregnancy outcome. 


\section{CASE SERIES}

\section{Case 1}

A 27-year-old female with history of spontaneous abortion of 9 weeks three years back and an intrauterine death at term two years back presented in OPD of Obstetrics and Gynecology Department of PBM Hospital, Bikaner for preconceptional checkup. In view of her past bad obstetric history, complete APLA profile was done. She was strongly positive for anti B2 glycoprotein $\operatorname{IgM}$ antibodies with values 29.35 (control <25) on 2 occasions 12 weeks apart.

She was diagnosed as primary APLA syndrome and after conception, she was put on aspirin $75 \mathrm{mg}$ once day (immediately after conception) and injection Enoxaparin 40 IU SC once a day (after confirming intrauterine pregnancy on USG) during entire pregnancy. She was also counselled about the maternal and fetal risks.

All routine blood investigations and USG were done. She visited ANC clinics regularly.
After completing 36 weeks of pregnancy aspirin and enoxaparin were withdrawn. Patient's routine investigations including coagulation profile were done and found to be normal. She was admitted with complaint of decreased fetal movement at 38 weeks of gestation and was taken up for caesarean section for fetal distress. She delivered a healthy baby boy of $2.7 \mathrm{~kg}$.

Her blood picture was normal during postnatal period.

A 32-year-old female presented to outpatient department (OPD) of Obstetrics and Gynecology Department of our hospital with history of one unexplained intrauterine death at term around 10 months back for prenatal counselling in view of her past pregnancy failure. Her APLA profile was done. She was found to be positive for lupus anticoagulant and negative for ANA, anti B2 glycoprotein 1 antibody and anticardiolipin antibody. The antibody titre continued to be positive even after 3 months. She was diagnosed as primary APS and conceived after 4 months. She was put on low dose aspirin $75 \mathrm{mg} \mathrm{OD}$, tablet Hydroxychloroquine $200 \mathrm{mg}$ HS and injection enoxaparin $40 \mathrm{mg}$ SC OD.

Table 1: Revised Sapparo Criteria for diagnosis of Antiphospholipid antibody syndrome (APS). ${ }^{5}$

Antiphospholipid antibody syndrome (APS) is present if at least one of the clinical criteria and one of the laboratory criteria that follow are met.

\section{Clinical criteria}

Vascular thromboses

One or more documented episodes of arterial, venous, or small vessel thrombosis - other than superficial venous

1. thrombosis - in any tissue or organ. Thrombosis must be confirmed by objective validated criteria. For histopathologic confirmation, thrombosis should be present without significant evidence of inflammation in the vessel wall.

2. Pregnancy morbidity

One or more unexplained deaths of a morphologically normal fetus at or beyond the 10th week of gestation, with

2.a normal fetal morphology documented by ultrasound or by direct examination of the fetus, or One or more premature births of a morphologically normal neonate before the 34th week of gestation because of:

2.b (i) eclampsia or severe pre-eclampsia defined according to standard definitions, or (ii) recognized features of placental insufficiency, or

Three or more unexplained consecutive spontaneous abortions before the 10th week of gestation, with maternal

2.c anatomic or hormonal abnormalities and paternal and maternal chromosomal causes excluded.

\section{Laboratory criteria}

These criteria for laboratory testing are consistent with current American Congress of Obstetricians and Gynecologists clinical management guidelines. ${ }^{20}$

Lupus anticoagulant (LA) present in plasma, on two or more occasions at least 12 weeks apart, detected

1. according to the guidelines of the International Society on Thrombosis and Haemostasis (Scientific Subcommittee on LAs/phospholipid dependent antibodies) ${ }^{5}$.

Anticardiolipin antibody (aCL) of IgG and/or IgM isotype in serum or plasma, present in medium or high titre

2. (i.e., $>40 \mathrm{GPL}$ or MPL, or $>$ the 99 th percentile), on two or more occasions, at least 12 weeks apart, measured by a standardized ELISA.

Anti-b2-glycoprotein-I antibody of IgG and/or IgM isotype in serum or plasma (in titre >the 99th percentile),

3. present on two or more occasions, at least 12 weeks apart, measured by a standardized ELISA, according to recommended procedures.

Investigators are strongly advised to classify APS patients in studies into one of the following categories: I, more than one laboratory criteria present (any combination); IIa, LA present alone; IIb, aCL present alone; IIc, anti- b2GPI antibody present alone 


\section{Case 2}

She took regular antenatal check-ups. These medications were withdrawn after 36 weeks of period of gestation.

Thereafter, all routine blood investigations and coagulation profile were done and found to be normal. Her prothrombin time was $13 \mathrm{sec}$ and INR was 1.015. LFT, RFT was also normal.

She delivered a healthy female child of $3.2 \mathrm{~kg}$ at 36 weeks of period of gestation through normal vaginal delivery. Her postnatal period was also uneventful.

\section{Case 3}

A 30-year-old female attended the OPD of Obstetrics and Gynecology Department with history of two spontaneous abortions at 6 and 12 weeks of gestation 3 years and 1.5 years back respectively. She also had history of high BP in her past pregnancy. Her complete APLA profile was done. ANA was positive but anti ds DNA, anti SM antibodies and RF were negative. Antiphospholipid (aPL) antibody (done by ELISA) was positive and remained so even after 12 weeks. Treatment was started with hydroxychloroquine, aspirin and prednisolone. She conceived 4 months after initiation of treatment. Her routine blood investigations and USG was done which were normal. All fetomaternal risks were explained. Inj. Enoxaparin 40mg SC was also started after confirming intrauterine pregnancy.

She was diagnosed with gestational hypertension at 32 weeks of gestation and was put on tablet labetalol 100mg BD orally. Drugs were withdrawn after 36 weeks of period of gestation. She was taken up for cesarean section for fetal distress and delivered a healthy male child of $3.1 \mathrm{~kg}$. Her postnatal period was uneventful.

\section{DISCUSSION}

Obstetric complications are the hallmark of antiphospholipid syndrome. The most common obstetric manifestation of this syndrome is recurrent miscarriage. About 5-15\% of recurrent pregnancy loss are associated with APS and $21-56 \%$ of SLE patients have secondary APS. ${ }^{3,4}$ The International Consensus Statement and American College of obstetricians and gynaecologists (ACOG) have given classification criteria for diagnosis of antiphospholipid syndrome (APS) which were revised in 2006 in Sapparo City, hence called revised Sapparo Criteria (Table 1). ${ }^{5}$

The pathophysiological mechanisms, underlying obstetric morbidity appear to be multiple, involving either thrombotic or non-thrombotic phenotypes of placenta. Also, the genetic and environmental factors influence the potential pathogenicity of these antibodies. The vascular thrombosis and pregnancy loss in APS is postulated to occur due to reduction in levels of Annexin V, a phospholipid binding protein with potent anticoagulant activity, on the placental villi of women with APS. ${ }^{6}$ The reduction of surface bound Annexin $\mathrm{V}$ is brought about by aPL antibodies.

Recently, direct trophoblastic damage by aPL, independent of mechanisms involving thrombosis and complement activation, has been demonstrated. Interaction of aPL with B2glycoprotein1, exposed during trophoblast syncytium formation, have been shown to cause inhibition of trophoblast invasiveness and this in turn contribute to early pregnancy loss.

Apart from fetal loss, current APS criteria include preterm delivery, oligohydramnios, neonatal complications (such as prematurity 930-60\%), fetal growth restriction (FGR), fetal distress and rarely fetal and neonatal thrombosis), associated maternal complications (like preeclampsia/eclampsia and HELLP syndrome and other aPL-related complications such as placental insufficiency) ${ }^{7-9}$ Screening of healthy pregnant women is not indicated. ${ }^{10}$

In patients with APS with pregnancy morbidity, gynecologic conditions such as uterine abnormality, hormonal imbalance like luteal phase defect, maternal and paternal karyotype abnormalities and presence of heritable procoagulant state, such as factor V leiden, should be ruled out as they may mimic APS. ${ }^{11}$

Another severe complication of pregnancy in case of APS is venous thromboembolism (VTE). Compared with nonpregnant women, there is 4-5 times higher risk of VTE in pregnant and postpartum women. ${ }^{12}$ When deep vein thrombosis (DVT) presents in pregnancy, there is predominance of left lower extremity clot formation due to compression of left common iliac vein by enlarging gravid uterus. $^{13}$

Majority of pregnant women with APS (70.5\%) will deliver a viable live infant with proper management. ${ }^{14}$ Ideally, these women should receive preconceptional counselling to outline the risks of pregnancy and appropriate management. Pregnancy should be discouraged in all women with pulmonary hypertension due to high risk of maternal death and should be postponed if woman has uncontrolled hypertension or recent thrombotic events, especially stroke. ${ }^{15}$

Proper counselling should be done in all cases regarding symptoms of thrombosis and frequent examination for sign or symptom of thrombosis or thromboembolism, severe preeclampsia or decreased fetal movement. We recommend frequent antenatal visits, every 2-4 weeks in 2nd trimester and 1-2 weeks in 3rd trimester. In patients with poor obstetric history, evidence of preeclampsia and growth retardation, ultrasonography is recommended every 3-4 weeks starting at 18-20-week gestation and fetal surveillance should begin at 32 weeks of gestation or 
earlier if uteroplacental insufficiency is suspected, and continue at least every week till delivery.

The main goal of treatment in pregnant women with APS is to improve maternal, fetal and neonatal outcome and minimising the risk of recognised complications including the need for iatrogenic preterm delivery. ${ }^{9}$

The evidence based clinical practice guidelines of American College of chest physicians suggest that women with antiphospholipid antibodies and a history of three or more early pregnancy losses or one or more late pregnancy losses who have no prior history of thrombosis, should receive treatment with combination aspirin and heparin (unfractionated or low molecular weight) during pregnancy. ${ }^{16}$ Aspirin $(81 \mathrm{mg} / \mathrm{dl})$ should be started as soon as woman conceives. Most investigators recommend preconceptional aspirin because of its possible beneficial effect on early stages of implantation. ${ }^{17}$ Unfractionated heparin (5000-10000 IU every 12 hours) or low molecular weight heparin in prophylactic doses (injection Enoxaparin 40mg SC every 24 hours) should be started after confirming a viable intrauterine pregnancy and continued till late in the third trimester. ${ }^{18}$

Patients should be advised to discontinue heparin if they suspect spontaneous labor. For induction or scheduled caesarean, prophylactic heparin should be discontinued at least 12 hours prior and restarted 6 to 8 hours following delivery or caesarean section.

In order to reduce the risk of postpartum DVT, antithrombotic coverage is recommended in all women with antiphospholipid syndrome with or without previous thrombosis. In patients with no previous thrombosis, the recommendation is prophylactic dose heparin or low molecular weight heparin therapy for 4-6 weeks after delivery and generally, women with thrombosis will need long term anticoagulation. ${ }^{16}$

Hydroxychloroquine directly inhibits the bound antiphospholipid antibody B2 glycoprotein-1 complex to phospholipid surfaces. ${ }^{19}$ Thus, it may be considered for an adjuvant antithrombotic role in patients with systemic lupus erythematosus (SLE) who are positive for antiphospholipid antibodies and also in patients with primary APS and recurrent thrombosis who have difficulty maintaining adequate anticoagulation intensity, or have a high-risk profile for major haemorrhage.

\section{CONCLUSION}

Diagnosis of antiphospholipid syndrome require a high index of suspicion during evaluation of women with recurrent pregnancy loss and vascular thrombosis. Obstetrician must check for anticardiolipin and lupus anticoagulant antibodies in women presenting with recurrent pregnancy loss. Management of APS is aimed to improve maternal and fetal outcome. Successful pregnancy can be possible with preconceptional counselling, proper selection of anticoagulants early in pregnancy with minimal risks to both mother and fetus and careful monitoring of pregnancy.

Funding: No funding sources

Conflict of interest: None declared

Ethical approval: Not required

\section{REFERENCES}

1. Hughes GR. Thrombosis, abortion, cerebral disease and the lupus anticoagulant. BMJ. 1983;287:1088-9.

2. Asherson RA, Khamashta MA, Ordi-Ros J, Derksen RH, Machin SJ, Barquinero J, et al. The "primary" antiphospholipid syndrome: major clinical and serological features. Medicine (Baltimore).1989;68(6):366-74.

3. Jerzak M, Wozniak S, Kotarski J. Pregnancy outcome in the woman with antiphospholipid antibody. Geburt Shilfe Frauenheilkd. 1996;56(8):410-3.

4. Galli M, Barbui T. Prevalence of different antiphospholipid antibody in Systemic Lupus Erythematosus and their relationship with the Antiphospholipid Antibody Syndrome. Clinical Chemistry. 2001;47(6):985-7.

5. Miyakis $\mathrm{S}$, Lockshin MD, Atsumi T. International consensus statement on an update of the classification criteria for definite antiphospholipid syndrome (APS). J Thromb Haemost. 2006;4:295-306.

6. Rand JH, Wu XX, Andree HAM, Lockwood CJ, Guller S, Scher J, et al. Pregnancy loss in the antiphospholipid antibody syndrome-a possible thrombogenic mechanism. $\mathrm{N}$ Engl J Med. 1997;337:154-60.

7. Wilson A, Gharavi AE, Koike T. International consensus statement on preliminary classification criteria for definite antiphospholipid syndrome. Arthritis Rheum. 1999;42:1309-11.

8. Avcin T, Cimaz R, Meroni PL. Recent advances in antiphospholipid antibodies and antiphospholipid syndromes in pediatric populations. Lupus. 2002;11:4-10.

9. Branch DW, Khamashta MA. Antiphospholipid syndrome: obstetric diagnosis, management, and controversies. Obstet Gynecol. 2003;101:1333-44.

10. Harris EN, Spinnato JA. Should anticardiolipin tests be performed in otherwise healthy pregnant women? Am J Obstet Gynecol. 1991;165:1272-7.

11. Gris JC, Quire I, Monpeyroux F. Case-control study of the frequency of thrombophilic disorders in couples with late foetal loss and no thrombotic antecedent - the Nimes obstetricians and haematologists study 5 (NOHA 5). Thrombosis Haemostasis. 1999;81:891-9.

12. Heit JA, Kobbervig CE, James AH. Trends in the incidence of venous thromboembolism during pregnancy or postpartum: a 30-year population-based study. Ann Intern Med. 2005;143:697-706.

13. Bourjeily G, Paidas M, Khalil H. Pulmonary embolism in pregnancy. Lancet. 2009. 
14. Bramham K, Hunt BJ, Germain S. Pregnancy outcome in different clinical phenotypes of antiphospholipid syndrome. Lupus. 2010;19:58-64.

15. Ruiz-Irastorza G, Khamashta MA. Lupus and pregnancy: ten questions and some answers. Lupus. 2008;17:416-20.

16. Bates SM, Greer IA, Pabinger I, Sofaer S, Hirsh J. Venous thromboembolism, thrombophilia, antithrombotic therapy, and pregnancy: American College of Chest Physicians Evidence-Based Clinical Practice Guidelines (8th edn). Chest. 2008;133:844S86S.

17. Carmona F, Font J, Azulay M. Risk factors associated with fetal losses in treated antiphospholipid syndrome pregnancies: a multivariate analysis. Am J Reprod Immunol. 2001;46:274-9.
18. Tincani A, Branch W, Levy RA. Treatment of pregnant patients with antiphospholipid syndrome. Lupus. 2003;12:524-9.

19. Rand J, Wu X, Quinn A, Chen P, Hathcock J, Taatjes D. Hydroxychloroquine directly reduces the binding of antiphospholipid antibody- $\beta 2$-glycoprotein I complexes to phospholipid bilayers. Blood. 2008;112:1687-95.

20. The American College of Obstetricians and Gynecologists. Antiphospholipid Syndrome. ACOG Practice Bulletin. 2011;118:1-8.

Cite this article as: Gaur P, Kochar S, Sharma V. A case series on successful pregnancy outcomes in patients with Apla syndrome. Int J Reprod Contracept Obstet Gynecol 2021;10:3575-9. 